\title{
Long Period Variables in the Magellanic Clouds: the OGLE/MACHO/2MASS/DENIS view
}

\author{
Martin Groenewegen \\ Instituut voor Sterrenkunde, Celestijnenlaan 200B, B-3001 Leuven, \\ Belgium
}

\begin{abstract}
I present a brief analysis of $K$-band period-luminosity relations for AGB stars in the SMC based on OGLE, 2MASS and DENIS data. I also present, for a selected sample of SMC and LMC stars, a comparison between the analysis of respective MACHO and OGLE data and a method to combine the data from the two surveys.
\end{abstract}

\section{Introduction}

In the course of the microlensing surveys in the 1990s, the monitoring of the Small and Large Magellanic Clouds has revealed an amazing number and variety of variable stars. Table 1 list the papers that deal primarily with variability related to red giants, Miras, semi-regulars and irregular variables.

Prior to 2003 the results focused on the LMC. The study presented here is an analysis of the (complete) OGLE database for the SMC and the combination with DENIS and 2MASS infrared data. Similar work has been carried out independently by Ita et al. (2003) and Kiss \& Bedding (2003).

Table 1. Surveys for red variables

\begin{tabular}{llll}
\hline Reference & Area & Number & Survey data \\
\hline Wood et al. 1999 \& & & & \\
Wood 2000 & $0.25 \mathrm{deg}^{2}$ LMC-bar & $1430 \mathrm{RV}^{1}$ & MACHO + IR \\
Cioni et al. 2001 & $0.5 \mathrm{deg}^{2}$ LMC-OC & $240 \mathrm{M}+\mathrm{SR}$ & EROS + D \\
Noda et al. 2002 & $14 \mathrm{deg}^{2}$ LMC & $146 \mathrm{LPV}$ & MOA + D \\
Lebzelter et al. 2002 & $0.25 \mathrm{deg}^{2}$ LMC-bar & $470 \mathrm{RV}$ & AGAPEROS + D \\
Cioni et al. 2003 & $0.25 \mathrm{deg}^{2}$ SMC & $458 \mathrm{RV}$ & MACHO + D $2 \mathrm{MASS}$ \\
Ita et al. 2003 & $1.0 \mathrm{deg}^{2}$ SMC-centre & $\sim 1800 \mathrm{RV}$ & OGLE + SIRIUS \\
Kiss \& Bedding 2003 & $4.5 \mathrm{deg}^{2}$ LMC-centre & $\sim 23000 \mathrm{RV}$ & OGLE + 2MASS \\
& & & with $J-K>0.9$ \\
\hline
\end{tabular}

$\overline{1 \text { red variables. }{ }^{2} \text { DENIS } I J K \text { survey. }{ }^{3} \text { A pre-selected sample of stars detected in an }}$ ISOCAM survey (Loup et al., in preparation.)

\section{The Model}

The procedure followed consists of two steps, the first being the fitting of the light curves. An iterative procedure is followed: (1) the best fit so far is 
subtracted from the data (in the first iteration this is the mean magnitude; (2) the Fourier transform is calculated to estimate the frequency; (3) a linear least-squares fitting routine is used to minimise the following function $I(t)=$ $I_{0}+\sum_{i=1}^{i=n_{\max }}\left(A_{\mathrm{i}} \sin \left(2 \pi t \omega_{i}\right)+B_{\mathrm{i}} \cos \left(2 \pi t \omega_{i}\right)\right)$; and (4) goto step (1). The stop condition is $n=3$, or when no significant frequency is found.

The second stage also consists of several steps: (1) selection of the candidate variable AGB star. (In the present paper for the SMC, $I<16.8$, plus any $P>50$ days, plus any amplitude $>0.05 \mathrm{mag}$ ); (2) correlation on position with the DENIS and 2MASS databases; (3) correlation with the SIMBAD database to eliminate known "non-AGB" stars (Cepheids, eclipsing binaries, etc.) and to obtain information on the M- or C-nature of the objects; and (4) visual inspection of the fit and corrective action if required.

This procedure leads to the identification of 975 objects, including 575 known carbon stars, 7 known oxygen-rich (M-) stars and no S-stars. The C/M ratio in the SMC is known to be large $(z 20)$, but the small number of confirmed M-stars is due to the small number of known M-stars with published accurate coordinates. The main difference with the other unbiased studies of the SMC by Ita et al. (2003) and Kiss \& Bedding (2003) is in the more restrictive choice here in period and amplitude selection which leads to a significantly smaller sample size than theirs. Another difference is that, in essentially all studies listed in Table 1 , the distinction between $\mathrm{C}$ - and M-stars is simply made on $(J-K)$ colour, while here this distinction is based on published carbon star surveys. Many (SMC) carbon stars have bluer colours than the usually adopted border line of 1.4 .

Of all the 68193 variable OGLE objects in the LMC and SMC, 50129 have a positional counterpart in the all-sky release of the 2MASS and 40793 with the DENIS survey. Studying samples of thousands of the least variable stars allows one to derive the following relations: $K_{2 \mathrm{MASS}}=K_{\text {DENIS }}+0.14, J_{2 \mathrm{MASS}}=$ $J_{\text {DENIS }}+0.08$ and $I_{\text {OGLE }}=I_{\text {DENIS }}+0.02$, with no colour term. This significant offset in $J K$ was from the 2MASS second incremental data release. It is likely due to an imperfect data reduction in the DENIS data reduction pipeline at that time (Cioni, private communication), as such an offset is not present when comparing DENIS and 2MASS data in the Galactic Bulge.

Fig. 1 shows the $K$-band period-luminosity relation for the full sample, and when an $I$-band amplitude cut of $0.45 \mathrm{mag}$ is imposed to simulate the classical Miras. For reference, the sequences ABCD adopted from Wood (2000) and Ita et al. (2003) have been plotted. The $K$-band used is on the 2MASS system. In particular, the DENIS photometry is corrected for as outlined above and also averaged with the 2MASS data, when available, to decrease the scatter due to the single epoch nature of the DENIS and 2MASS surveys. Fig. 2 shows colourmagnitude and colour-colour diagrams showing that many known C-stars have blue $(J-K)$ colours.

\section{Comparison between MACHO and OGLE}

Having so much data that is mostly used in a statistical sense, one can ask the question whether periods derived from MACHO and OGLE data agree on a star-by-star basis. In order to study this I have made a comparison with 

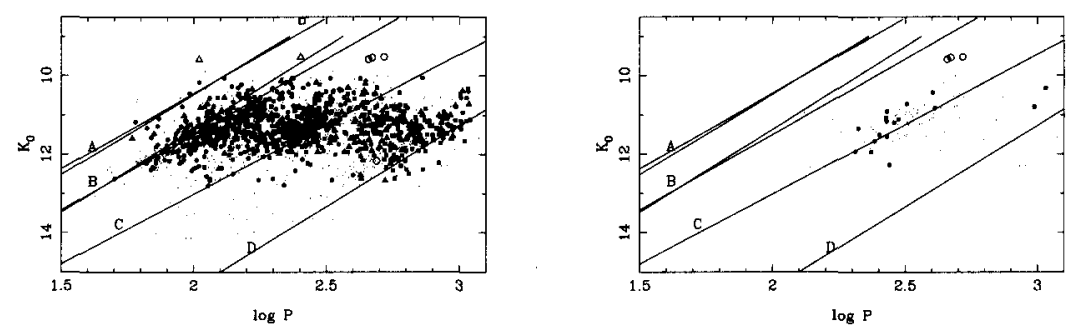

Figure 1. $\quad K$-band period-luminosity relation in the SMC, for all stars in the sample (left panel) and for those stars with an $I$-band amplitude $>0.45 \mathrm{mag}$ (right panel). Sequences $\mathrm{ABCD}$ have been indicated, adopted from Wood (2000) and Ita et al. (2003). Known C-stars are indicated by a filled symbol, known M-stars by an open symbol and others by a dot.

selected SMC objects in common with Cioni et al. (2003) and with selected LMC objects with $P<25 \mathrm{~d}$ that are on sequence $\mathrm{A}$ in common with Wood (2000). This involved downloading the respective MACHO data and analysing it the same way as I described before. This allows then to compare the quoted periods with those I derive from the OGLE and the MACHO data. Space does not permit to show any examples, but they can be found in my presentation which is available at http://www.ster.kuleuven.ac.be/ $\sim$ groen. In many cases the agreement is good, but in some $50 \%$ of the cases differences of some form occur. They can be related to: how many periods are searched for and how many are actually quoted in the literature; or, the period quoted is found, but not as the first (most significant) by me; or, I find more periods than quoted. For example, for most of the objects I investigated in common with Wood, he finds only one period (although he looked for up to three), while I often find three. The additional periods would fall on sequences $B$ and $D$ and therefore seem to be real. This indicates one of the uncertainties involved in this type of analysis: where to put the limit on what is a significant frequency and what is not. In rarer cases, the data itself is "flawed" in some sense and that leads to different periods.

If the MACHO "red" and OGLE $I$ data can be described to first order like $R(t)=M_{\mathrm{R}}+A_{\mathrm{R}} \cos \left(\omega_{\mathrm{R}} t+\phi_{\mathrm{R}}\right)$ and $I(t)=M_{\mathrm{I}}+A_{\mathrm{I}} \cos \left(\omega_{\mathrm{I}} t+\phi_{\mathrm{I}}\right)$ and if there is no phase or frequency shift with colour then one expects: $R(t)-I(t)=$ $\left(1-A_{\mathrm{I}} / A_{\mathrm{R}}\right) R(t)+\left(M_{\mathrm{R}}-M_{\mathrm{I}}\right)$. For every MACHO data point in a dataset it was verified if there was a OGLE data point within a time $\Delta t$ and if more than one, the closest in time was kept. Taking $\Delta t=(0.1-0.3) \mathrm{d}$ gives $>40$ points. Outliers are removed. A linear fit of $(R-I)$ versus $R$ is performed and outliers removed. Then the MACHO data is converted to $I$ and joined with the OGLE data to form a new dataset, on which the period analysis is performed. This seems to give satisfactory results in most cases and examples can again be found in my presentation.

Acknowledgments. This publication makes use of data products from the Two Micron All Sky Survey, which is a joint project of the University of Mas- 

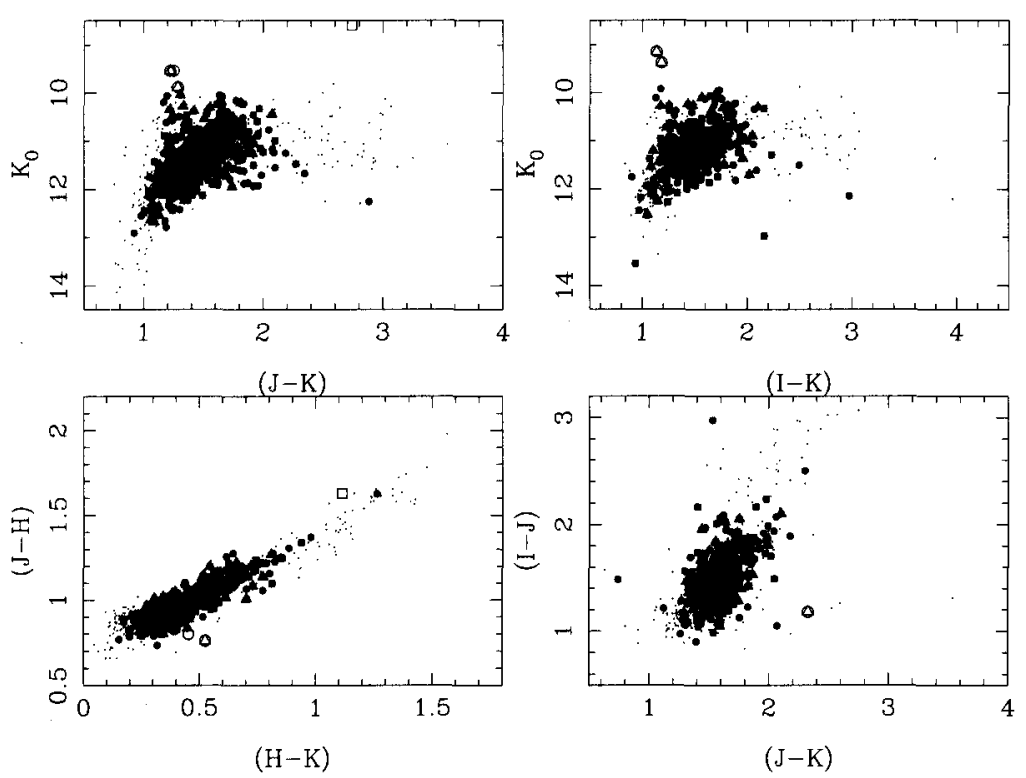

Figure 2. Colour-magnitude and colour-colour diagrams for the SMC. Symbols as in Fig. 1.

sachusetts and the Infrared Processing and Analysis Center/California Institute of Technology, funded by NASA and the NSF. This paper utilises public domain data obtained by the MACHO Project, jointly funded by the US Department of Energy through the University of California, Lawrence Livermore National Laboratory under contract No. W-7405-Eng-48, by the National Science Foundation through the Center for Particle Astrophysics of the University of California under cooperative agreement AST-8809616 and by the Mount Stromlo and Siding Spring Observatory, part of the Australian National University.

\section{References}

Cioni M.-R. et al. 2001, A\&A 377, 495

Cioni M.-R.L. et al. 2003, A\&A, 406, 51

Ita Y. et al. 2003, preprint

Kiss L.L., Bedding T. 2003, MNRAS, 343, L79

Lebzelter T., Schulteis M., Melchior A.L. 2002, A\&A, 393, 573

Noda S. et al. 2002, MNRAS 330, 137

Wood P.R. et al. 1999, in IAU Symposium 191, Asymptotic Giant Branch Stars, eds. T. Le Bertre, A. Lebre \& C. Waelkens, (ASP, San Francisco), 151

Wood P.R. 2000, PASA, 17, 18 